\title{
Linear Inequalities Among Graph Invariants: Using GraPHedron to Uncover Optimal Relationships
}

\author{
Julie Christophe, Sophie Dewez, Jean-Paul Doignon, Gilles Fasbender, Philippe Grégoire, \\ David Huygens, and Martine Labbé \\ Département de Mathématique, Université Libre de Bruxelles, Boulevard du Triomphe, CP 216, \\ B-1050, Brussels, Belgium
}

Sourour Elloumi

CEDRIC, Conservatoire National des Arts et Métiers, Paris, France

Hadrien Mélot

Institute d'Informatique, Université de Mons-Hainaut, Mons, Belgium

\author{
Hande Yaman \\ Department of Industrial Engineering, Bilkent University, Bilkent, Turkey
}

\begin{abstract}
Optimality of a linear inequality in finitely many graph invariants is defined through a geometric approach. For a fixed number of graph vertices, consider all the tuples of values taken by the invariants on a selected class of graphs. Then form the polytope which is the convex hull of all these tuples. By definition, the optimal linear inequalities correspond to the facets of this polytope. They are finite in number, are logically independent, and generate precisely all the linear inequalities valid on the class of graphs. The computer system GrapHedron, developed by some of the authors, is able to produce experimental data about such inequalities for a "small" number of vertices. It greatly helps in conjecturing optimal linear inequalities, which are then hopefully proved for any number of vertices. Two examples are investigated here for the class of connected graphs. First, all the optimal linear inequalities for the stability number and the number of edges are obtained. To this aim, a problem of Ore (1962) related to the Turán Theorem (1941) is solved. Second, several optimal inequalities are established for three invariants: the maximum degree, the irregularity, and the diameter. (C) 2008 Wiley Periodicals, Inc. NETWORKS, Vol. 52(4), 287-298 2008
\end{abstract}

Keywords: graph invariants; polytope; optimal linear inequalities; GraPHedron

Received September 2005; accepted February 2008

Correspondence to: J.-P. Doignon; e-mail: doignon@ulb.ac.be

Contract grant sponsor: Communauté française de Belgique - Actions de Recherche Concertées.

DOI 10.1002/net.20250

Published online 3 June 2008 in Wiley InterScience (www.interscience. wiley.com).

C 2008 Wiley Periodicals, Inc.

\section{INTRODUCTION}

An important research stream in Graph Theory studies relationships among graph invariants. Invariants are numerical indices which summarize the graph structure: their values are preserved by isomorphisms. Relations among invariants range from very easy (e.g., the sum of vertex degrees equals twice the number of edges) to very deep and difficult (like many results in extremal graph theory). During the last two decades, several computer based systems have been developed in order to generate new relations among invariants. Some of them even produced conjectures in an automated way; see [39] for a survey emphasizing contributions to algebraic graph theory. Here is a brief sketch of the main existing systems (among others):

- the GRAPH system by Cvetković et al. [18-23] interactively computes invariants and includes also a theorem-proving component;

- the INGRID system of Brigham and Dutton [8-11] implements manipulation of formulae involving graph invariants;

- the Graffiti system of Fajtlowicz et al. [25-31] generates a priori conjectures and then eliminates those that are rejected by a database of counter-examples or are not interesting;

- the AutoGraphix system of Caporossi and Hansen [2, $12-15,24,32,35,36,40,41]$ generates extremal or nearextremal graphs for some relations on graph invariants, then derives conjectures either automatically or with the user's intervention.

Hansen [38] divides such computer systems into two classes: automated systems which provide conjectures in a 
fully automated way (i.e., without human intervention apart from the problem statement), and computer-assisted systems otherwise. The new system we have implemented is a computer-assisted one.

The investigation of relations among graph invariants should tackle the following two general questions: when is a relation interesting? when is it optimal? Here, we derive answers to these questions using a polyhedral approach in which graphs are represented as points in a space of invariants. This approach is quite natural but has not yet been exploited in a systematic way. The system AutoGraphix of Caporossi and Hansen uses three different methods to derive conjectures automatically. Among them is a "geometric approach" which consists of considering "extremal graphs as points in a space of characteristics, then uses a convexhull (or gift-wrapping) algorithm to find facets, which correspond to conjectures" ([15], p. 83). However, as Caporossi and Hansen consider larger instances than we do here, they explore only a selection of graphs which are extremal or near-extremal for a given objective function and they only study the facets relevant for the corresponding optimization problem (minimization or maximization). On the contrary we consider all nonisomorphic graphs of a selected class and characterize all the facets of the convex hull. As we now explain, the method always singles out a finite number of optimal relations from which all other relations follow.

Given a fixed set of $p$ invariants for graphs, linear inequalities among these invariants are of particular interest. Following our general approach, we consider all linear inequalities which hold for graphs from a given class $\mathcal{C}$ (e.g., the class of connected graphs with $n$ vertices). Each graph $G$ from the class $\mathcal{C}$ is represented by some point in the $p$-dimensional real space $\mathbb{R}^{p}$ : the point has coordinates equal to the values taken by the $p$ invariants for $G$. The convex hull of all the resulting points (obtained when $G$ varies in $\mathcal{C}$ ) is the polytope of graph invariants for the selected set of invariants and the class $\mathcal{C}$ of graphs. ${ }^{1}$ Any linear inequality on $\mathbb{R}^{p}$ valid for the polytope of graph invariants induces a linear relationship among the invariants under investigation, which holds for the class $\mathcal{C}$. Furthermore, such an inequality can be considered as best possible exactly when it defines a facet of the polytope. The reason is twofold: all other linear inequalities among the invariants are logical consequences (more specifically, are dominated by a positive combination) of the facet defining inequalities, and no facet defining inequality can be a logical consequence of other such inequalities. Besides, these facet defining inequalities are finite in number; we will refer to them as the optimal inequalities. Notice also that a description by optimal inequalities of the polytope of graph invariants can be of great help when a linear combination of the invariants has to be maximized over the graphs in the class $\mathcal{C}$. We remark in passing that the above approach also applies to nonlinear relationships among invariants: it suf-

\footnotetext{
${ }^{1}$ For ease of exposition, we assume that the polytope is full dimensional, i.e., of dimension $p$.
}

fices to associate coordinates to, say, powers or products of invariants.

The polyhedral interpretation of the linear inequalities among the $p$ selected graph invariants also suggests a fruitful strategy to formulate conjectures. The first three stages can be implemented on a computer. First, for $n$ not too large, generate all the graphs on $n$ vertices which belong to the class $\mathcal{C}$ under study (the program geng [43] can be instrumental, possibly supplemented with ad hoc routines). Second, compute for each graph the values taken by the graph invariants. Third, for any $n$ in the admissible range, determine in $\mathbb{R}^{p}$ the convex hull of the resulting $p$-dimensional points (running for instance the software porta [16] or cdd [33]). Fourth, interpret the facet defining inequalities which were obtained for the various values of $n$. The strategy helps in conjecturing linear inequalities among the selected invariants. There remains to mathematically establish these relationships in full generality for the class $\mathcal{C}$. Two applications of the strategy are provided in this paper.

The method can be used to verify whether known inequalities among invariants are the best possible in a very strong sense. On the contrary, it is common practice to show that a given inequality among invariants is tight by exhibiting an instance for which the relation holds with equality. This argument shows only that the inequality defines a supporting hyperplane of the convex hull defined above. It does not establish that the inequality defines a facet. This last property, according to our approach, singles out the optimal linear inequalities.

In the next section, we provide a brief description of the computer system GraPHedron designed to support our investigations (see Mélot [44] for technical details). A web interface to the system is publicly available at http: / / www.graphedron. net. One of the distinctive features of GraPHedron is the automatic production of a report summarizing information on the polytope of graph invariants, such as the list of extreme points, the list of facets, drawings of graphs (just one, or all) which produce any given extreme point, etc.

Apart from a brief section containing general definitions, the rest of the paper illustrates our approach with results obtained in two cases for the class of connected graphs. First, we completely treat a case of two invariants, namely the stability number and the number of edges. Our system produced figures of the resulting polytope for the number of graph vertices up to 11 . We were then able to establish the complete list of facet defining inequalities for all values of $n$. On the way, we solve a problem raised by Ore [45] (see Problem 1 of Section 13.4) in relation to the Turán Theorem and thus improve recent results of Harant and Schiermeyer [42]. The problem asks for the least number of edges in a connected graph on $n$ vertices with stability number $\alpha$. Turán [46] solved a similar problem for general graphs by describing nonconnected optimal solutions. The system GraPHedron was essential for us to solve Ore's problem: we understood from its outputs the variety of critical graphs that a proof should take into account. 
A second case is meant to illustrate intricacies. Three invariants are considered: the maximum degree, the diameter and the irregularity. Although several optimal linear inequalities are obtained, determining the complete list of all such inequalities remains an open problem.

\section{OUTLINE OF THE SYSTEM GraPHEDRON}

The main steps in using the system GraPHedron are as follows.

\subsection{Problem Statement}

The user selects a class $\mathcal{C}$ of graphs (e.g., connected graphs, triangle-free graphs, trees, ...) and a set of invariants (e.g., number of edges, diameter, maximum degree, ...). Notice that new invariants can easily be added to the system.

\subsection{Data Generation}

Using geng [43], the system generates all the nonisomorphic graphs in $\mathcal{C}$ with a fixed number of vertices $n$, and stores them (to avoid re-generation of the same class in the future). Of course, it generates the graphs only for "small" values of $n$ (the number of values of $n$ can be increased if the class of graphs is more restricted; see [44] for details). Then, for each selected invariant, the system computes and stores its values for all the graphs which were generated. Storing the values is useful especially when the computations of the invariant take a long time (actually, computing invariants is the most time-consuming part of the whole process; for details, see Mélot [44]).

\subsection{Determination of Optimal Linear Inequalities Between Invariants}

For each $n$, all the different points, i.e., distinct tuples of values of the invariants, are formed. Then, their convex hull is computed as a list of the facets and a list of the extreme points. Currently the programs cdd [33] or porta [16] can be used as subroutines to compute the convex hull. However there exist many software packages implementing different convex hull algorithms (see [3] for a comparison of the most prevalent algorithms and for computational experiments).

\subsection{Interactive Visualization of the Results and Derivation of Conjectures}

Finally, the user has to study the inequalities. He may interact with the system to see and/or print the graphs corresponding to a certain extreme point. In the case of two invariants, the automatic report produced by the system contains a drawing of the polygon of graph invariants. Optional information can be required in the report, e.g., statistics about an invariant's values or representation of the distribution of points in the polytopes.

\section{NOTATION}

Let $G=(V, E)$ be a connected graph with vertex set $V$ and edge set $E$. As usual, the number of vertices of the graph $G$ is denoted by $n$ and the number of edges by $m$. A set $A$ of vertices of $G$ is stable if $\{v, w\} \notin E$ for all $v, w \in A$. The maximum cardinality of a stable set of $G$ is the stability number $\alpha(G)$. We denote by $d_{v}$ the degree of the vertex $v$, that is $d_{v}=|\{w \in V:\{v, w\} \in E\}|$, and by $\Delta(G)$ the maximum degree $\max _{v \in V} d_{v}$. The distance between two vertices $v$ and $w$ is the length of a shortest path from $v$ to $w$. The diameter $D(G)$ is the maximum distance between two vertices of $G$. A diameter path in $G$ is a path of length $D(G)$. The irregularity of $G$ is $\iota(G)=\sum_{\{v, w\} \in E}\left|d_{v}-d_{w}\right|$.

A staris a tree with one vertex adjacent to all other vertices. Other classical graphs will also be used, such as the complete graph $K_{n}$, the complete bipartite graph $K_{a, b}$, the path $L_{n}$, and the cycle $C_{n}$. Additional graphs are introduced when needed.

A polytope is the convex hull conv $S$ of a finite set $S$ of points in $\mathbb{R}^{d}$. For terminology about polytopes, we generally follow Ziegler [47].

\section{STABILITY NUMBER AND NUMBER OF EDGES}

The general methodology outlined in Section 1 is applied here to the case of two invariants, the stability number and the number of edges. Fixing the number $n$ of vertices, we associate to any connected graph $G=(V, E)$ on $n$ vertices the pair $(\alpha, m)$ where, as before, $\alpha=\alpha(G)$ and $m=|E|$. Our goal is to determine, for any $n$, all facet defining inequalities for the convex polygon

$$
\begin{aligned}
P_{\alpha, m}^{n}= & \operatorname{conv}\left\{(\alpha, m) \in \mathbb{Z}^{2}:\right. \text { there exists a connected } \\
& \operatorname{graph} G=(V, E) \text { with }|V|=n, \alpha(G)=\alpha,|E|=m\} .
\end{aligned}
$$

The polygon $P_{\alpha, m}^{n}$ lies in the plane $\mathbb{R}^{2}$ with coordinates $x_{\alpha}$ and $x_{m}$. Figure 1 illustrates the case for $n=10$.

Figure 1, together with similar figures for other values of $n$, suggests grouping the facets of $P_{\alpha, m}^{n}$ in three families (in the present case, the facets are one-dimensional). These families respectively consist of: (i) one horizontal facet lying on the line $x_{m}=n-1$; (ii) facets to the right, forming a path from the extreme point $(n-1, n-1)$ to the extreme point $\left(1,\left(\begin{array}{c}n \\ 2\end{array}\right)\right)$; (iii) facets to the left, connecting the extreme point $\left(\left\lfloor\frac{n+1}{2}\right\rfloor, n-1\right)$ to the extreme point $\left(1,\left(\begin{array}{l}n \\ 2\end{array}\right)\right)$. Our analysis will treat these three families one after the other, and establish in each case the complete list of facets together with the corresponding facet defining inequalities. To avoid trivialities, we will assume $n \geq 4$ in this section.

\subsection{The Horizontal Facet}

Since we consider only connected graphs on $n$ vertices, the minimum number of edges in such a graph equals $n-1$, a value which arises exactly for trees. The stability number of such trees varies from $\left\lfloor\frac{n+1}{2}\right\rfloor$ (for a path on $n$ vertices) to $n-1$ (for a star $K_{1, n-1}$ ). These observations result in a 


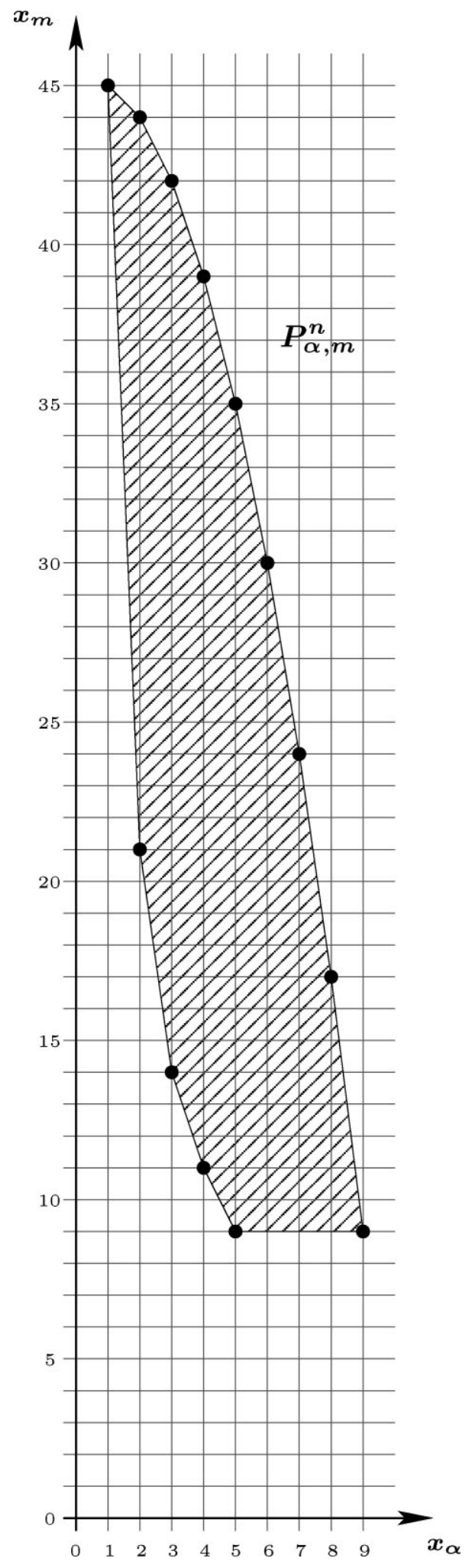

FIG. 1. The polygon $P_{\alpha, m}^{n}$ for $n=10$.

horizontal facet for $P_{\alpha, m}^{n}$, with extreme points $\left(\left\lfloor\frac{n+1}{2}\right\rfloor, n-1\right)$ and $(n-1, n-1)$. The corresponding facet defining inequality is of course $x_{m} \geq n-1$.

\subsection{The Rightmost Facets}

The following proposition concerns the second family of facets.

Proposition 1. For $k=1,2, \ldots, n-2$, the inequality

$$
k x_{\alpha}+x_{m} \leq\left(\begin{array}{c}
n-k \\
2
\end{array}\right)+k n
$$

defines a facet of $P_{\alpha, m}^{n}$ with extreme points

$$
\begin{aligned}
\left(k,\left(\begin{array}{c}
n-k \\
2
\end{array}\right)+k(n-k)\right) & \text { and } \\
& \left(k+1,\left(\begin{array}{c}
n-k \\
2
\end{array}\right)+k(n-k-1)\right) .
\end{aligned}
$$

All facets and extreme points of $P_{\alpha, m}^{n}$ to the right of some point of $P_{\alpha, m}^{n}$ are of these types.

Thus the extreme points on the rightmost part of $P_{\alpha, m}^{n}$ form the sequence $\left(k,\left(\begin{array}{c}n-k \\ 2\end{array}\right)+k(n-k)\right)$ for $k=1,2, \ldots, n-1$, which starts at $\left(1,\left(\begin{array}{l}n \\ 2\end{array}\right)\right)$ and ends at $(n-1, n-1)$.

Proof. We first establish that (1) is valid for each extreme point of $P_{\alpha, m}^{n}$. With $\alpha$ denoting the stability number of a graph $G=(V, E)$ for which $|V|=n$ and $|E|=m$, this amounts to proving

$$
m \leq\left(\begin{array}{c}
n-k \\
2
\end{array}\right)+k(n-\alpha) .
$$

We proceed by considering two cases.

(a) Case $k \leq \alpha$. In some stable set $S$ of maximum size in $G$, select any subset $T$ of $k$ vertices. The number of edges disjoint from $T$ is at most $\left(\begin{array}{c}n-k \\ 2\end{array}\right)$. Any other edge has exactly one vertex in $T$; its other vertex lies outside $S$. The number of such edges is at most $k(n-\alpha)$. Summing up, we get inequality (3).

(b) Case $k>\alpha$. Select a set $U$ of $k$ vertices which contains a maximum-size stable set $S$. Any edge is either (i) disjoint from $U$, or (ii) formed by one vertex in $S$ and the other one in $V \backslash S$, or (iii) formed by one vertex in $U \backslash S$ and the other one in $V \backslash S$. Thus the total number of edges is at most $\left(\begin{array}{c}n-k \\ 2\end{array}\right)+$ $\alpha(n-\alpha)+(k-\alpha)(n-\alpha)$, from which (3) follows.

We thus have proved that (1) holds for $P_{\alpha, m}^{n}$.

Each of the two points given in (2) comes from at least one connected graph, namely the graph having as edges all those pairs of vertices not included in a fixed subset of $k$, resp. $k+1$, vertices. As is easily checked, these two points satisfy (1) with equality, and the same holds for a second linear inequality also valid for the polytope. Consequently, they are the extreme points of a facet defined by (1). Moreover, there cannot be any other facet because we have found extreme points with abscissas increasing from 1 to $n-1$ by steps of 1.

We point out that another proof of Proposition 1 exists, in which the first step consists of characterizing the extreme 
points of $P_{\alpha, m}^{n}$. This characterization can be established in two steps: (i) determining the maximum number of edges in a connected graph with a given number of vertices and given stability number; (ii) showing that the resulting points in $\mathbb{R}^{2}$ are convexly independent. The easy solution to (i) states that the maximum number of edges equals $(n-\alpha)(n+\alpha-1) / 2$. As this function of $\alpha$ is strictly concave, (ii) becomes trivial. Inequality (1) can then be derived. This line of argument produces also a nonlinear inequality attributed in [37] to Tomescu:

$$
\alpha \leq\left\lfloor\frac{1}{2}+\sqrt{\frac{1}{4}+n(n-1)-2 m}\right\rfloor .
$$

\subsection{The Leftmost Facets}

For the third and last family of facets, we will first infer the corresponding extreme points by answering the following question: what is the minimum number of edges in connected graphs with a fixed number of vertices and a fixed stability number? This question is listed as an open problem in Ore [45]. A complete answer is contained in Proposition 3 below. It constitutes the variant for connected graphs of a famous result of Turán [46], which we now recall. For given integer numbers $n$ and $\alpha$ satisfying $n \geq \alpha \geq 1$, the Turán graph $T(n, \alpha)$ has $n$ vertices and is the disjoint union of $\alpha$ cliques with balanced sizes (i.e., sizes equal to $\left\lfloor\frac{n}{\alpha}\right\rfloor$ or $\left\lceil\frac{n}{\alpha}\right\rceil$; the last two expressions give the same value if and only if $\alpha$ divides $n)$. We let $t(n, \alpha)$ denote the number of edges in the Turán graph $T(n, \alpha)$.

Proposition 2. [46]. Any graph on $n$ vertices with stability number $\alpha$ has at least $t(n, \alpha)$ edges. Moreover, this graph has exactly $t(n, \alpha)$ edges if and only if it is (isomorphic to) the Turán graph $T(n, \alpha)$.

Thus the only graph achieving the minimum number of edges in Proposition 2 has $\alpha$ connected components. Of course, by adding $\alpha-1$ carefully selected edges, we obtain various connected graphs on $n$ vertices with stability number $\alpha$. Notice that in the case where $n=2 \alpha+1$, odd cycles on $n$ vertices still provide other examples with the same values of $\alpha$ and $m$. Despite their diversity (as illustrated by outputs from GraPHedron), we are able to prove that all these connected graphs have the minimum possible number of edges for given $n$ and $\alpha$. The proof is more elaborate than the simple ones known for the Turán result (and presented, e.g., in Bollobás [6]). Also, it covers more cases than the connected one (which is obtained in Proposition 3 below for $c=1$, see Corollary 1).

Proposition 3. Any graph $G$ on $n$ vertices with stability number $\alpha$ and with $c$ connected components has at least $t(n, \alpha)+\alpha-c$ edges. The lower bound is tight in all cases.

For $1 \leq c \leq \alpha \leq n$, let $f_{c}(n, \alpha)$ be the minimum number of edges for graphs satisfying the conditions in
Proposition 3.

To prove $f_{c}(n, \alpha)=t(n, \alpha)+\alpha-c$, we first establish two lemmas (a third one comes later).

Lemma 1. Suppose $n \geq 2 \alpha$. If $f_{1}(n, \alpha)=t(n, \alpha)+\alpha-1$, then for $c=1,2, \ldots, \alpha$, we have $f_{c}(n, \alpha)=t(n, \alpha)+\alpha-c$.

Proof (of Lemma 1). The assumption $n \geq 2 \alpha$ implies that each maximal clique of the Turán graph $T(n, \alpha)$ has more than one vertex. By adding $\alpha-c$ carefully selected edges to $T(n, \alpha)$, we see

$$
t(n, \alpha)+\alpha-c \geq f_{c}(n, \alpha),
$$

and similarly by adding $c-1$ carefully selected edges to a graph achieving $f_{c}(n, \alpha)$,

$$
f_{c}(n, \alpha)+c-1 \geq f_{1}(n, \alpha) .
$$

Thus

$$
t(n, \alpha)+\alpha-c \geq f_{c}(n, \alpha) \geq f_{1}(n, \alpha)-(c-1) .
$$

By our assumption, the first and last expressions in (4) are equal. The conclusion follows.

Lemma 2. For $1 \leq \beta \leq \alpha \leq n$, we have

$$
t(n, \beta) \geq t(n, \alpha)+\alpha-\beta .
$$

Proof (of Lemma 2). We only need to consider the case where $\alpha=\beta+1$. To transform $T(n, \beta+1)$ into $T(n, \beta)$, we pick a clique of size $\left\lfloor\frac{n}{\beta+1}\right\rfloor$ in the first graph, delete its vertices and add $\left\lfloor\frac{n}{\beta+1}\right\rfloor$ vertices in all to the other cliques. Clearly, the number of edges increases at each deletion/addition of a single vertex.

Proof (of Proposition 3). By adding $\alpha-c$ selected edges to $T(n, \alpha)$, we see that inequality $f_{c}(n, \alpha) \leq t(n, \alpha)+\alpha-c$ holds. Hence, only the following inequality remains to be proved:

$$
f_{c}(n, \alpha) \geq t(n, \alpha)+\alpha-c .
$$

Assume first $n<2 \alpha$. Then $t(n, \alpha)=n-\alpha$ because the Turán graph then consists of $2 \alpha-n$ isolated vertices plus $n-\alpha$ parallel edges. On the other hand, any graph on $n$ vertices which has $c$ connected components has at least $n-c$ edges. This results in $f_{c}(n, \alpha) \geq n-c=t(n, \alpha)+\alpha-c$, which gives (6).

Assume now $n \geq 2 \alpha$. By Lemma 1, we only need to establish the result for $G$ connected. It remains to be proved that

$$
f_{1}(n, \alpha) \geq t(n, \alpha)+\alpha-1 .
$$

We proceed by recurrence on the number $n$ of vertices. Let $S$ be a maximum stable set of $G$, and let $b$ be the number of connected components of $G \backslash S$ (thus $b \leq \alpha$ ). By Lemma 3 below, the number of edges in the cut $\delta(S)$ is at least $n-\alpha+$ $b-1$. On the other hand, $V \backslash S$ induces in $G$ a graph with 
$b$ components and stability number $\beta$ satisfying $\beta \leq \alpha<$ $n-\alpha$. By the induction assumption together with Lemma 1 , the graph induced by $V \backslash S$ has at least $f_{b}(n-\alpha, \beta)=$ $t(n-\alpha, \beta)+\beta-b$ edges. Summing up, we get

$$
m \geq n-\alpha+b-1+t(n-\alpha, \beta)+\beta-b
$$

and then by Lemma 2

$$
\begin{aligned}
m & \geq n-\alpha+b-1+t(n-\alpha, \alpha)+\alpha-\beta+\beta-b \\
& =n-\alpha+t(n-\alpha, \alpha)+\alpha-1 \\
& =t(n, \alpha)+\alpha-1
\end{aligned}
$$

The last equality directly follows from the structure of Turán graphs: the addition of a vertex to each of the $\alpha$ maximal cliques of $T(n-\alpha, \alpha)$ produces $T(n, \alpha)$. Inequality (7) is thus proved.

The last assertion in Proposition 3 is correct in view of the examples provided just before the statement of this proposition.

Lemma 3. As in the proof of Proposition 3, let $G=(V, E)$ be a connected graph with $n$ vertices, $m$ edges and stability number $\alpha$. Take a maximum stable set $S$ in $G$, and let $C_{1}$, $C_{2}, \ldots, C_{b}$ be the $b$ connected components of $G \backslash S$. Then the cut $\delta(S)$ satisfies

$$
|\delta(S)| \geq n-\alpha+b-1
$$

Proof (of Lemma 3). For $i=1,2, \ldots, b$, build a spanning tree in $C_{i}$. Then extend the union of these $b$ (sub)trees into a spanning tree $T$ of $G$. Clearly, $E(T) \cap \delta(S)$ is an acyclic set of edges of $G$ which covers $S$ and at least one vertex in each $C_{i}$ (possibly several vertices in a given $C_{i}$ ). Denote by $G^{\prime}$ the graph $(V, E(T) \cap \delta(S))$ and let $A_{1}, A_{2}, \ldots, A_{\ell}$ be the connected components with more than one vertex of $G^{\prime}$. For $j=1,2, \ldots, \ell$, denote by $s_{j}$ (resp. $r_{j}$ ) the number of vertices of $A_{j}$ in $S$ (resp. not in $S$ ). Thus, the subgraph of $G^{\prime}$ induced by $A_{j}$ is a tree with $s_{j}+r_{j}$ vertices (see Fig. 2 for an illustration).
For any fixed $j$, two vertices in $A_{j} \backslash S$ cannot belong to the same $C_{i}$ (otherwise there would be a cycle formed by edges of the tree $T$ ). We derive

$$
\sum_{j=1}^{\ell}\left(r_{j}-1\right)=b-1
$$

by repeatedly collapsing vertices as follows. Let $A_{j}$ and $A_{j^{\prime}}$ each have a vertex in the same $C_{i}$. Then by collapsing these two vertices we collect $A_{j}$ and $A_{j^{\prime}}$ together while leaving the left-hand side of (8) unchanged because $\left(r_{j}-1\right)+\left(r_{j^{\prime}}-1\right)=$ $\left(r_{j}+r_{j^{\prime}}-1\right)-1$. Once all such collapsings have been done, the left-hand side of (8) contains only one term which equals $b-1$ (because exactly one vertex remains in each of the $b$ sets $C_{i}$ ).

We now establish a lower bound on the number $p_{j}$ of edges of $G$ connecting the $r_{j}$ vertices in $A_{j} \backslash S$ to any vertices of $S$. By connectivity of $A_{j}$ in the graph $G^{\prime}$, we surely have $p_{j} \geq s_{j}+r_{j}-1$. Moreover $A_{j} \backslash S$ is stable in $G$ (because as shown before $A_{j} \backslash S$ cannot have two vertices in the same $C_{i}$ ), so there must be at least $r_{j}$ vertices in the maximum stable set $S$ which are adjacent in $G$ to at least one vertex in $A_{j} \backslash S$. Then $p_{j} \geq\left(r_{j}+s_{j}-1\right)+\left(r_{j}-s_{j}\right)=2 r_{j}-1$.

Consider now any vertex $u$ of $G$ not covered by $E(T) \cap$ $\delta(S)$, thus $u \notin S$. As $S \cup\{u\}$ cannot be stable, there is some edge connecting $u$ to $S$.

Finally, any vertex outside $S$ is either covered by $E(T) \cap$ $\delta(S)$ (and then, for some $j$, belongs to $A_{j}$ ) or is not covered (and then is adjacent to some vertex in $S$ ). In addition, using $p_{j} \geq 2 r_{j}-1$ and (8), we get

$$
\begin{aligned}
|\delta(S)| & \geq\left(\sum_{j=1}^{\ell}\left(2 r_{j}-1\right)\right)+\left(n-\alpha-\sum_{j=1}^{\ell} r_{j}\right) \\
& =n-\alpha+\sum_{j=1}^{\ell}\left(r_{j}-1\right)=n-\alpha+b-1 .
\end{aligned}
$$

The most important case in Proposition 3 occurs when $c=1$. In view of the following explicit form of the Turán number (see, e.g., [5])

$$
t(n, \alpha)=\left(\left\lceil\frac{n}{\alpha}\right\rceil-1\right) \cdot\left(n-\frac{\alpha}{2}\left\lceil\frac{n}{\alpha}\right\rceil\right),
$$

we thus have proved the next result.

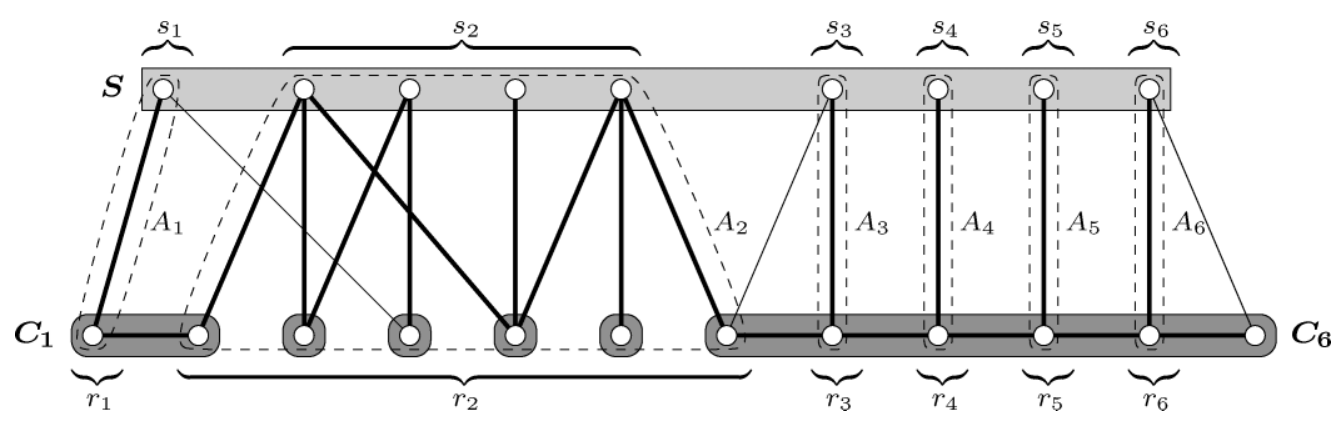

FIG. 2. An example of graph $G$ for the proof of Lemma 3. The edges in $E(T)$ are shown in bold lines. 
Corollary 1. The minimum number of edges for all connected graphs on $n$ vertices with stability number $\alpha$ equals

$$
\left(\left\lceil\frac{n}{\alpha}\right\rceil-1\right) \cdot\left(n-\frac{\alpha}{2}\left\lceil\frac{n}{\alpha}\right\rceil\right)+\alpha-1 .
$$

Corollary 1 improves the main result in [42], which consists of a lower bound on the minimum number of edges. After we submitted this work for publication, other proofs of Corollary 1 were independently found by Gitler and Valencia [34], and also by Bougard and Joret [7] who use Brooks' Theorem. The latter authors provide additional results, including the variant of Corollary 1 for 2-connected graphs and a characterization of minimum graphs for both the connected and 2 -connected cases. Finding the minimum number of edges for $k$-connected graphs when $k \geq 3$ remains an open problem.

Coming back to the polytope $P_{\alpha, m}^{n}$, we now determine its leftmost extreme points and facets. We take advantage of the following points of $P_{\alpha, m}^{n}$ delivered by Corollary 1:

$$
p_{k}=(k, t(n, k)+k-1), \quad \text { for } k=1,2, \ldots,\left\lfloor\frac{n+1}{2}\right\rfloor .
$$

A first step is to show that all of these points are boundary points. Next, we determine which of them are extreme. The facets then follow immediately (see Corollary 2 below).

Proposition 4. The extreme points of the leftmost part of the boundary of $P_{\alpha, m}^{n}$ are exactly the points provided in (11) for $k$ in $\left\{2,3, \ldots,\left\lfloor\frac{n-1}{2}\right\rfloor\right\}$ satisfying

$$
\left\lceil\frac{n}{k-1}\right\rceil \neq\left\lfloor\frac{n}{k+1}\right\rfloor+1
$$

and also for $k=1$ and $k=\left\lfloor\frac{n+1}{2}\right\rfloor$.

Proof. Leaving aside the extreme points $\left(1,\left(\begin{array}{c}n \\ 2\end{array}\right)\right)$ and $\left(\left\lfloor\frac{n+1}{2}\right\rfloor, n-1\right)$, we assume $1<k<\left\lfloor\frac{n+1}{2}\right\rfloor$ in the rest of the proof. To establish that the point $p_{k}$ is on the boundary of $P_{\alpha, m}^{n}$, it suffices to show that the "Turán function" (for a fixed value of $n$ )

$$
t(n,): \quad\left\{1,2, \ldots,\left\lfloor\frac{n+1}{2}\right\rfloor\right\} \rightarrow \mathbb{R}: \quad x \mapsto t(n, x)
$$

is the restriction of a convex function from $\left[1,\left\lfloor\frac{n+1}{2}\right\rfloor\right]$ to $\mathbb{R}$ (notice that we may discard the term $k-1$ which appears in the second coordinate of $p_{k}$, because it does not alter convexity). In turn, we need only prove for $k=2,3, \ldots,\left\lfloor\frac{n-1}{2}\right\rfloor$

$$
t(n, k) \leq \frac{1}{2}(t(n, k-1)+t(n, k+1)) .
$$

Inequality (14) follows from the following three assertions (again for $k=2,3, \ldots,\left\lfloor\frac{n-1}{2}\right\rfloor$ ): The line with equation

$$
x_{m}-t(n, k)=-\left(\begin{array}{c}
\left\lfloor\frac{n}{k}\right\rfloor+1 \\
2
\end{array}\right)\left(x_{\alpha}-k\right)
$$

1. goes through the point $p_{k}=(k, t(n, k))$,

2. supports the point $p_{k-1}=(k-1, t(n, k-1))$, and

3 . supports the point $p_{k+1}=(k+1, t(n, k+1))$.

The first assertion is clear. Setting $s=\left\lfloor\frac{n}{k}\right\rfloor$, we see that the second one is equivalent to

$$
t(n, k-1)-t(n, k) \geq\left(\begin{array}{c}
s+1 \\
2
\end{array}\right) .
$$

We derive the latter inequality by checking how many new edges are created when the Turán graph $T(n, k)$ is transformed into the similar graph $T(n, k-1)$. As explained before Proposition 2, the graph $T(n, k)$ is a union of cliques with size equal to $s$ and possibly also $s+1$. To transform it into $T(n, k-1)$, we select a clique with size $s$ and move its vertices one after the other to other cliques. At the first step, we delete $s-1$ edges in the clique, and gain at least $s$ edges in the augmented clique; in all, we gain at least one edge. For the second vertex, we have a gain of at least 2 (because we lose $s-2$ edges this time), etc. Thus the total number of edges increases by at least $1+2+\cdots+s$, that is $\left(\begin{array}{c}s+1 \\ 2\end{array}\right)$.

Now the third assertion about the line in (15) translates into

$$
t(n, k)-t(n, k+1) \leq\left(\begin{array}{c}
s+1 \\
2
\end{array}\right) .
$$

The graph $T(n, k+1)$ is a union of $k+1$ cliques, of size say $d$ and (possibly also) $d+1$. To transform $T(n, k+1)$ into $T(n, k)$, we move all vertices of some clique of size $d$ to other cliques. As we can create cliques only of size $s$ or $s+1$ (because of the structure of $T(n, k)$ ), the number of edges increases by at most $s-(d-1)$ at the first move, then at most $s-(d-2)$, etc.; at the last move, we gain at most $s$ edges. Hence the increase in the number of edges is bounded above by $\frac{1}{2} d(2 s+1-d)$. For $s$ fixed, this quantity is maximized for $d=s$ or $d=s+1$. We conclude that Assertion 3 is correct.

Having thus shown that all points $p_{k}$ provided in (11) are on the boundary of $P_{\alpha, m}^{n}$, we notice next that all leftmost extreme points of $P_{\alpha, m}^{n}$ must be among the $p_{k}$ 's (because two successive points $p_{k}$ have their abscissas differing by 1 ). Also, a point $p_{k}$ is not extreme if and only if the line in (15) goes also through the points $p_{k-1}$ and $p_{k+1}$, which happens exactly if both inequalities (16) and (17) are satisfied with equality. By inspecting the arguments which led to these inequalities, it is easily seen that both equalities occur if and only if the sizes of the maximal cliques in $T(n, k-1)$, resp. $T(n, k+1)$, are all also sizes of maximal cliques in $T(n, k)$. This is equivalent to saying that $n /(k+1)$ and $n /(k-1)$ lie in an interval with endpoints equal to two consecutive integers. Finally, $p_{k}$ is not extreme if and only if $\left\lceil\frac{n}{k-1}\right\rceil=\left\lfloor\frac{n}{k+1}\right\rfloor+1$.

Example. For $n=24$, the values of $k$ which do not provide an extreme point are 7 and then 9, 10, 11. In particular, all points $p_{9}, p_{10}$ and $p_{11}$ lie on the segment joining the extreme points $p_{8}$ and $p_{12}$. 
TABLE 1. The optimal linear inequalities in $\alpha$ and $m$ for connected graphs.

\begin{tabular}{ll}
$m \geq n-1$ & \\
$k \alpha+m \leq\left(\begin{array}{c}n-k \\
2\end{array}\right)+k n$ & for $k=1,2, \ldots, n-2$ \\
$m-t(n, k)-(k-1) \geq$ & for $k=2,3, \ldots,\left\lfloor\frac{n+1}{2}\right\rfloor$ with \\
$\quad(t(n, k)-t(n, k-1)+1)(\alpha-k)$ & $\left\lceil\frac{n}{k-1}\right\rceil \neq\left\lfloor\frac{n}{k+1}\right\rfloor+1$ \\
\hline
\end{tabular}

Corollary 2. The leftmost facets of $P_{\alpha, m}^{n}$ are defined by the following inequalities:

$x_{m}-t(n, k)-(k-1) \geq(t(n, k)-t(n, k-1)+1)\left(x_{\alpha}-k\right)$

forvalues $k=2,3, \ldots,\left\lfloor\frac{n+1}{2}\right\rfloor$ satisfying $\left\lceil\frac{n}{k-1}\right\rceil \neq\left\lfloor\frac{n}{k+1}\right\rfloor+1$.

We have completed our analysis of the case with the two invariants $\alpha$ and $m$. The full list of optimal linear inequalities appears in Table 1. The polygon $P_{\alpha, m}^{n}$ has an interesting property which is not shared by all similar polytopes constructed for other choices of invariants: Any point from $P_{\alpha, m}^{n}$ which has integer coordinates is produced by some graph in the class considered (here, the class of connected graphs on $n$ vertices).

\section{MAXIMUM DEGREE, IRREGULARITY, AND DIAMETER}

Our methodology is now applied to the following three invariants of a connected graph $G$ : the maximum degree $\Delta(G)$, the irregularity $\iota(G)$ and the diameter $D(G)$.

Let us quickly provide a motivation for the irregularity. A graph $G$ is regular if all the degrees of its vertices are equal, otherwise $G$ is irregular. Clearly, it is of interest to design a measure of how much a graph $G$ is irregular. Among the measures of irregularity proposed in the literature, the most prevalent ones are: the Collatz-Sinogowitz index which is the difference between the largest eigenvalue of the adjacency matrix and the average degree [17]; the variance of degrees [4]; and the irregularity $\iota(G)$ of Albertson [1], which we use here. Note that all these indices are equal to zero if $G$ is regular. Tight upper bounds in terms of $n$ and $m$ are given in [4] for the Collatz-Sinogowitz index and the variance of degree. For the most recent invariant $\iota(G)$ a tight upper bound in term of $n$ is given in [1]. Another bound in terms of $n$ and $m$ is given in [41] when $G$ is general and in [35] when $G$ is a chemical tree, i.e., a tree with a maximum degree equal to 4 . However, to the best of our knowledge, there are no other results concerning the irregularity $\iota(G)$ of Albertson. Consequently, it is worth studying this invariant in relation to other parameters than the number of edges. We choose the maximum degree and the diameter which express simple but different characteristics of graphs.
The polytope of graph invariants is here

$$
\begin{aligned}
& P_{\Delta, l, D}^{n}=\operatorname{conv}\left\{(\Delta, \iota, D) \in \mathbb{Z}^{3}:\right. \text { there exists a } \\
& \text { connected graph } G=(V, E) \text { with }|V|=n, \\
& \Delta(G)=\Delta, \iota(G)=\iota \text { and } D(G)=D\} .
\end{aligned}
$$

For any fixed $n$, the polytope $P_{\Delta, l, D}^{n}$ lies in the 3-dimensional space $\mathbb{R}^{3}$ with coordinates $x_{\Delta}, x_{\iota}$ and $x_{D}$. As shown in the next proposition, it is full-dimensional. Consequently, the facets of $P_{\Delta, l, D}^{n}$ are its two-dimensional faces.

Proposition 5. Let $P_{\Delta, l, D}^{n}$ be the polytope defined by (18). If $n \geq 4$, then $\operatorname{dim}\left(P_{\Delta, l, D}^{n}\right)=3$.

Proof. To exhibit four affinely independent points lying in $P_{\Delta, l, D}^{n}$, consider the following graphs on $n$ vertices: the complete graph $K_{n}$, the cycle $C_{n}$, the star $K_{1, n-1}$, and the path $L_{n}$. The corresponding points in $\mathbb{R}^{3}$ are shown below:

$$
\begin{aligned}
K_{n}: & (n-1,0,1), \\
C_{n}: & (2,0,\lfloor n / 2\rfloor), \\
K_{1, n-1}: & (n-1,(n-1)(n-2), 2), \\
L_{n}: & (2,2, n-1) .
\end{aligned}
$$

It can easily be seen that they are affinely independent.

The output produced by GraPHedron for $n=4$, $5, \ldots, 10$ led us to conjecture that (under various assumptions) five families of linear inequalities are facet defining. We proceed to give the corresponding proofs.

Proposition 6. If $n \geq 4$, the inequality

$$
x_{\Delta} \leq n-1
$$

is facet defining for $P_{\Delta, l, D}^{n}$.

Proof. The validity of (19) for $P_{\Delta, l, D}^{n}$ is obvious. Because the origin does not belong to the affine plane $H \equiv$ $x_{\Delta}=n-1$, it is now sufficient to exhibit three linearly independent vectors of $P_{\Delta, l, D}^{n}$ belonging to $H$. Let us consider the following three graphs: the complete graph $K_{n}$, the star $K_{1, n-1}$, and finally $G_{3}$, a star augmented with one edge. The corresponding vectors, namely

$$
\begin{aligned}
K_{n}: & (n-1,0,1), \\
K_{1, n-1}: & (n-1,(n-1)(n-2), 2), \\
G_{3}: & (n-1, n(n-3), 2),
\end{aligned}
$$

belong to $H \cap P_{\Delta, l, D}^{n}$ and are linearly independent.

Proposition 7. The inequality

$$
x_{\Delta}+x_{D} \leq n+1
$$

is facet defining for $P_{\Delta, l, D}^{n}$ when $n \geq 4$. 
Proof. (i) We first prove that $\Delta(G)+D(G) \leq n+1$ holds for any connected graph $G$ on $n$. Take a diameter path $P$ of $G$, thus $P$ is a shortest path $v_{1}, v_{2}, \ldots, v_{D(G)+1}$ (with length $D(G)$ ). Let $v^{*}$ be a vertex having degree $\Delta(G)$ and let $\mathcal{N}\left(v^{*}\right)$ be the closed neighborhood of $v^{*}$, i.e., $\mathcal{N}\left(v^{*}\right)=\{v \in$ $\left.V:\left\{v, v^{*}\right\} \in E\right\} \cup\left\{v^{*}\right\}$. From

$$
\begin{gathered}
|P|+\left|\mathcal{N}\left(v^{*}\right)\right|-\left|P \cap \mathcal{N}\left(v^{*}\right)\right| \leq n, \\
|P|=D(G)+1 \text {, and }\left|\mathcal{N}\left(v^{*}\right)\right|=\Delta(G)+1 \text {, we derive } \\
\Delta(G)+D(G)+2-\left|P \cap \mathcal{N}\left(v^{*}\right)\right| \leq n .
\end{gathered}
$$

It remains to prove $\left|P \cap \mathcal{N}\left(v^{*}\right)\right| \leq 3$.

1. Suppose $P \cap \mathcal{N}\left(v^{*}\right)=\varnothing$. Then $\left|P \cap \mathcal{N}\left(v^{*}\right)\right|=0 \leq 3$.

2. Suppose $P \cap \mathcal{N}\left(v^{*}\right) \neq \varnothing$ and $v^{*} \notin P$. Then $\mid P \cap$ $\mathcal{N}\left(v^{*}\right) \mid \leq 3$, otherwise $P$ could not be a shortest path.

3. Suppose $P \cap \mathcal{N}\left(v^{*}\right) \neq \varnothing$ and $v^{*}=v_{k} \in P$. If $1<k<$ $D(G)+1$, then $P \cap \mathcal{N}\left(v^{*}\right)=\left\{v_{k-1}, v^{*}, v_{k+1}\right\}$ because $P$ is a shortest path, and so $\left|P \cap \mathcal{N}\left(v^{*}\right)\right|=3$. If $v^{*}=v_{1}$ or $v^{*}=v_{D(G)+1}$, then $\left|P \cap \mathcal{N}\left(v^{*}\right)\right|=2$.

(ii) Having proved that (20) is valid for $P_{\Delta, l, D}^{n}$, we now show that it defines a facet. The path $L_{n}$, the star $K_{1, n-1}$, and the star augmented with one edge, denoted as $G_{3}$ in the proof of Proposition 6, produce the vectors

$(2,2, n-1), \quad(n-1,(n-1)(n-2), 2), \quad(n-1, n(n-3), 2)$.

These three vectors satisfy (20) with equality and, when $n \geq$ 4 , they are linearly independent.

Let $H=(V(H), E(H))$ be a subgraph of $G=$ $(V, E)$. We define the irregularity along $H$ as $\iota(H)=$ $\sum_{\{k, l\} \in E(H)}\left|d_{k}-d_{l}\right|$ where the degree $d_{k}$ of the vertex $k$ is relative to the graph $G$. To establish in Proposition 8 the validity of our next inequality, we first improve inequality (20).

Lemma 4. Let $G=(V, E)$ be a connected graph such that $\iota(G)<\Delta(G)$. Then $\Delta(G)+D(G) \leq n$.

Proof. We show for any connected graph $G$ that $\Delta(G)+$ $D(G)>n$ implies $\iota(G) \geq \Delta(G)$. By (20), we may assume $\Delta(G)+D(G)=n+1$. From the proof of Proposition 7, any vertex of maximum degree must be on some diameter path. Let $P=v_{1}, v_{2}, \ldots, v_{D(G)+1}$ be a diameter path containing at least one vertex of degree $\Delta(G)$.

By construction, the diameter path $P$ contains $D(G)+1$ vertices. Consequently, $n-(D(G)+1)=\Delta(G)-2$ vertices do not belong to $P$. We call them exterior vertices (relatively to the diameter path $P$ ) and denote them by $w_{1}, w_{2}, \ldots, w_{\Delta(G)-2}$. The extremities $v_{1}$ and $v_{D(G)+1}$ of the diameter path $P$ are of degree at most $\Delta(G)-1$. So, let $v^{*}=v_{k} \in P \backslash\left\{v_{1}, v_{D(G)+1}\right\}$ be the vertex of $P$ with maximum degree such that the subpath $P_{1} \subset P$ which joins $v_{1}$ to $v^{*}$ does not contain any other vertex of maximum degree. There are (a)

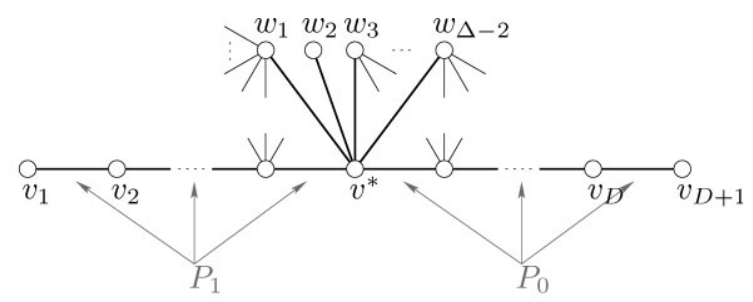

(b)

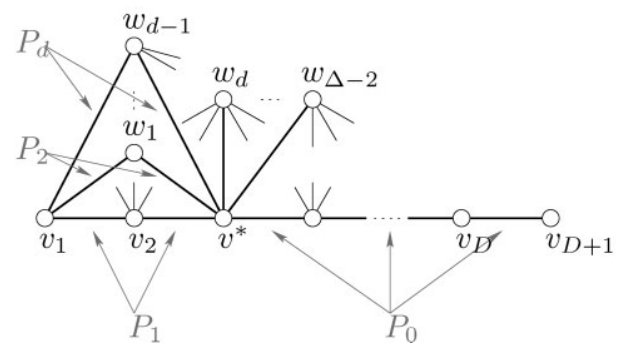

FIG. 3. All the exterior vertices are adjacent to the vertex $v^{*}$. In Case (a), one has $d_{v_{1}}=1$, while in Case (b), one has $1<d_{v_{1}} \leq \Delta-1$.

$\Delta(G)$ edges incident to the vertex $v^{*}$ and two of them belong to the diameter path $P$. Thus the $\Delta(G)-2$ remaining edges are incident to the $\Delta(G)-2$ exterior vertices. We consider two cases (see Fig. 3).

Case (a). Suppose $d_{v_{1}}=1$. By comparing the degrees along $P_{1}$, we obtain for the irregularity along $P_{1}$

$$
\iota\left(P_{1}\right) \geq \Delta(G)-1
$$

Case (b). Suppose now $d_{v_{1}}=d>1$. (Note that the distance between $v_{1}$ and $v^{*}$ cannot be greater than two; in other terms, $v^{*}=v_{2}$ or $v^{*}=v_{3}$.) We have

$$
\iota\left(P_{1}\right) \geq \Delta(G)-d .
$$

All the vertices adjacent to $v_{1}$, but one, are exterior vertices. Let $P_{2}, P_{3}, \ldots, P_{d}$ be the paths defined by $P_{r}=v_{1}, w_{r-1}, v^{*}$ (after relabelling the exterior vertices if necessary). Because the paths $P_{1}, P_{2}, \ldots, P_{d}$ have no common edge, it follows that

$$
\sum_{r=1}^{d} \iota\left(P_{r}\right) \geq d(\Delta(G)-d) .
$$

Moreover, $2 \leq d \leq \Delta(G)-1$, which implies

$$
d(\Delta(G)-d) \geq \Delta(G)-1 \text {. }
$$

This proves

$$
\sum_{r=1}^{d} \iota\left(P_{r}\right) \geq \Delta(G)-1 .
$$

Now in both Cases (a) and (b) let $P_{0}$ be the subpath of $P$ joining $v^{*}$ to $v_{D(G)+1}$. Clearly, the irregularity along $P_{0}$ is at least 1 , and so

$$
\iota(G) \geq \Delta(G)
$$


Proposition 8. The inequality

$$
n x_{\Delta}-x_{\iota}+(n-1) x_{D} \leq n^{2}-1
$$

is valid for $P_{\Delta, l, D}^{n}$. If $n \geq 5$ and $n$ is odd, the inequality is facet defining.

Proof. (i) We first prove the validity of (22). For a graph $G=(V, E)$, this inequality can be rewritten as

$$
\Delta(G)+D(G) \leq n+1+\frac{\iota(G)-\Delta(G)}{n-1} .
$$

If $\Delta(G) \leq \iota(G)$, inequality (23) is dominated by inequality (20) and is thus valid. If $\Delta(G)>\iota(G)$, then Lemma 4 gives $\Delta(G)+D(G) \leq n$, which implies (23) because $\Delta(G) \leq n-1$.

(ii) We now prove that, for $n \geq 5$ and $n$ odd, inequality (22) is facet defining. The following three graphs produce vectors which are linearly independent and satisfy (22) with equality: $K_{n}, L_{n}$, and the graph on $n$ vertices such that one of its vertices has degree $n-1$ and the $n-1$ other ones have degree $n-2$. Notice that this last graph only exists for $n$ odd.

On the contrary, as we will see at the end of this section, inequality (22) is not facet defining when $n$ is even. Before considering two more inequalities, we establish two lemmas.

Lemma 5. Let $G$ be a connected graph with $n \geq 4, D(G) \geq$ 3 and $\Delta(G)+D(G)=n+1$. Then $\iota(G) \geq 2 \Delta(G)-2$.

Proof. We build upon the proof of Lemma 4. Let $P=v_{1}$, $v_{2}, \ldots, v_{D(G)+1}$ be a diameter path containing some vertex $v^{*}$ of degree $\Delta(G)$. As in the proof of Lemma 4 , we get $v^{*} \in\left\{v_{2}\right.$, $\left.v_{3}, \ldots, v_{D(G)}\right\}$. Let $V^{\prime}$ be the set of exterior vertices. Note that an exterior vertex cannot be adjacent to both $v_{1}$ and $v_{D(G)+1}$, because of our assumption $D(G) \geq 3$. Thus, $V^{\prime}$ is the disjoint union of the subset $V_{1}$ of exterior vertices adjacent to $v_{1}$, the subset $V_{2}$ of exterior vertices adjacent to $v_{D(G)+1}$, and the subset $V^{\prime \prime}$ of all remaining exterior vertices. Consider three cases.

(a) Suppose $V_{1} \neq \varnothing \neq V_{2}$. (This is possible only if $D(G)=3$ or 4 because $\Delta(G)+D(G)=n+1$ implies that all exterior vertices are adjacent to $v^{*}$.) Consider the set $\mathcal{P}_{1}$ of paths $v_{1}, t, v^{*}$ with $t \in V_{1}$, and the set $\mathcal{P}_{2}$ of paths $v_{D(G)+1}, u, v^{*}$ with $u \in V_{2}$. The same argument as in the proof of

\section{Lemma 4.}

shows that the total irregularity computed along the paths from $\mathcal{P}_{1}$, or from $\mathcal{P}_{2}$, must be at least $\Delta(G)-1$. Because $V_{1} \cap V_{2}=\varnothing$, it is clear that no two paths in $\mathcal{P}_{1} \cup \mathcal{P}_{2}$ have a common edge. Thus

$$
\iota(G) \geq 2 \Delta(G)-2 .
$$

(b) Suppose $V_{1} \neq \varnothing$ and $V_{2}=\varnothing$ (the case where $V_{1}=\varnothing$ and $V_{2} \neq \varnothing$ can be treated along the same line). This implies $d_{v_{D(G)+1}}=1$. Similar to Case (a), the total irregularity along paths $v_{1}, t, v^{*}$ with $t \in V_{1}$ is at least $\Delta(G)-1$. Furthermore, the path $Q=v_{D(G)+1}, v_{D(G)}, \ldots, v^{*}$ has no common edge with those paths and it satisfies $\iota(Q) \geq \Delta(G)-1$. Hence

$$
\iota(G) \geq 2 \Delta(G)-2 .
$$

(c) Suppose $V_{1}=\varnothing=V_{2}$. Then

$$
\begin{aligned}
\iota(G) & \geq \iota\left(\left\{v_{1}, v_{2}, \ldots, v^{*}\right\}\right)+\iota\left(\left\{v^{*}, \ldots, v_{D(G)}, v_{D(G)+1}\right\}\right) \\
& \geq 2 \Delta(G)-2 .
\end{aligned}
$$

Lemma 6. Let $G$ be a connected graph with $n$ even, $n \geq 4$, $D(G)=2$, and $\Delta(G)=n-1$. Then

$$
\iota(G) \geq 2 n-4 \text {. }
$$

Proof. Let $k \geq 1$ be the number of vertices having degree $n-1$. The edges connecting the vertices of degree $n-1$ to vertices of smaller degree lead to

$$
\iota(G) \geq k(n-k) .
$$

If $2 \leq k \leq n-2$, inequality (24) follows from inequality (25). Because the graph cannot be complete, the case $k=n-1$ is impossible. We still have to consider the case where $k=1$.

Suppose $k=1$. Let $v$ be the unique vertex of degree $n-1$ and let $\ell$ denote the number of vertices of degree $n-2$. The contribution to $\iota(G)$ of the edges connecting the vertex $v$ to the $\ell$ vertices of degree $n-2$ is $\ell$, and the contribution to $\iota(G)$ of the edges connecting the vertex $v$ to the $n-\ell-1$ vertices of degree lower than $n-2$ is at least $2(n-\ell-1)$. Finally, the edges connecting the $\ell$ vertices of degree $n-2$ to the $n-\ell-1$ vertices of degree lower than $n-2$ contribute to $\iota(G)$ at least $\ell(n-\ell-2)$. Summing up, we obtain

$$
\iota(G) \geq(\ell+2)(n-\ell-1) .
$$

If $0 \leq \ell \leq n-3$, inequality (24) follows from the above inequality. Notice that $\ell=n-1$ is impossible since $n$ is even. Thus the result for $k=1$ and $\ell=n-2$ remains to be proved.

Suppose $k=1$ and $\ell=n-2$. Then, there exists a unique vertex of degree $d<n-2$ and

$$
\begin{aligned}
\iota(G) & \geq(n-2)+(n-1-d)+(d-1)(n-2-d) \\
& =n-2+d(n-2-d)+1
\end{aligned}
$$

Because $d(n-2-d) \geq n-3$ for $1 \leq d \leq n-3$, the expression above leads to inequality (24).

Proposition 9. The inequalities

$$
2 x_{\Delta}-x_{\iota}+2 x_{D} \leq 2 n
$$

and

$$
2(n-1) x_{\Delta}-x_{\iota}+2(n-2) x_{D} \leq 2\left(n^{2}-n-1\right)
$$

are facet defining for $P_{\Delta, l, D}^{n}$ when $n$ is even and $n \geq 4$. 


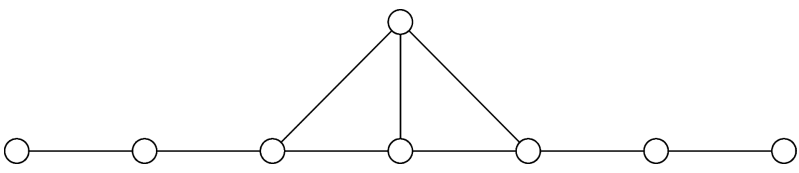

FIG. 4. The boat on 8 vertices.

Proof. (i) The validity of inequality (28) can easily be proved: we show $2 \Delta(G)-\iota+2 D \leq 2 n$ for any connected graph $G$. If $G$ is regular, then (28) reduces to $\Delta(G)+D(G) \leq$ $n$, which is always valid by Lemma 4 . If $G$ is not regular, then $\iota(G) \geq 2$ and inequality (28) is dominated by $2 \Delta(G)+$ $2 D(G) \leq 2 n+2$, which is true by Proposition 7 .

The validity of (29) can be shown using similar arguments as in the proof of Proposition 7. Indeed, for a connected graph $G$, inequality (29) is equivalent to

$$
\Delta(G)+D(G) \leq n+1+\frac{2+(\iota(G)-2 \Delta(G))}{2(n-2)} .
$$

Consider two cases:

(a) If $\Delta(G)+D(G) \leq n$, then (30) holds because $\Delta(G) \leq$ $n-1$ and $\iota \geq 0$ imply that the right-hand side is bounded from below by $n$.

(b) Suppose $\Delta(G)+D(G)=n+1$. Then the fact that the right-hand side is bounded from below by $n+1$ follows from Lemmas 5 and 6.

(ii) We now prove that the two inequalities in the statement are facet defining. For (29), three linearly independent vectors giving equality are provided by the graphs $K_{n}, L_{n}$ and the "boat" on $n$ vertices, i.e., the graph consisting of the path $L_{n-1}$ with an exterior vertex adjacent to its three consecutive middle vertices (see Fig. 4). This last graph is well defined for $n \geq 4$ and $n$ even.

For (28), replace the boat with the graph sketched in Figure 5. This graph is defined for $n \geq 6$ and it has five vertices of degree 3 , one vertex of degree 1 , and all other vertices of degree 2. In case $n=4$, use rather the complete graph minus one edge.

Notice that the sum of inequalities (28) and (29) is equal to twice (22). Consequently, the latter inequality does not define a facet for $n$ even.

In this section we have established some of the optimal inequalities for the diameter, the irregularity and the maximum degree of a connected graph. Even if we are not able at this time to provide a complete description of the polytope of graph invariants, we have still shown that our method leads to nontrivial and interesting results. The problem of finding all

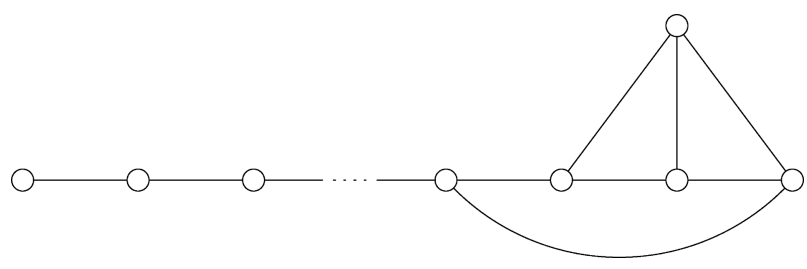

FIG. 5. A graph used in the proof of Proposition 9. optimal linear inequalities for the diameter, the irregularity and the maximum degree is left open.

\section{ACKNOWLEDGMENTS}

The authors thank the editor and two referees for a careful reading of their manuscript, Romina Hites for help with the English language, and Alain Hertz for a comment on one of their proofs.

\section{REFERENCES}

[1] M. Albertson, The irregularity of a graph, Ars Combin 46 (1997), 219-225.

[2] M. Aouchiche, G. Caporossi, and P. Hansen, Variable neighborhood search for extremal graphs 8. Variations on graffiti 105, Congr Numer 148 (2001), 129-144.

[3] D. Avis, D. Bremmer, and R. Seidel, How good are convex hull algorithms? Comput Geom 7 (1997), 265-301.

[4] F. Bell, A note on the irregularity of graphs, Linear Algebra Appl 161 (1992), 45-54.

[5] C. Berge, Graphes, Dunod, Paris, 1983.

[6] B. Bollobás, Graph theory, Springer, New York, 1979.

[7] N. Bougard and G. Joret, Turán's theorem and k-connected graphs, J Graph Theory 58 (2008), 1-13.

[8] R. Brigham and R. Dutton, Ingrid: A software tool for extremal graph theory research, Congr Numer 39 (1983), 337-352.

[9] R. Brigham and R. Dutton, A compilation of relations between graph invariants, Networks 15 (1985), 73-107.

[10] R. Brigham and R. Dutton, A compilation of relations between graph invariants. Suppl 1, Networks 21 (1991), 421-455.

[11] R. Brigham, R. Dutton, and F. Gomez, Ingrid: A graph invariant manipulator, J Symbolic Comput 7 (1989), 163-177.

[12] G. Caporossi, D. Cvetković, I. Gutman, and P. Hansen, Variable neighborhood search for extremal graphs 2. Finding graphs with extremal energy, J Chem Inf Comput Sci 39 (1999), 984-996.

[13] G. Caporossi, I. Gutman, and P. Hansen, Variable neighborhood search for extremal graphs 4 . Chemical trees with extremal connectivity index, Comput Chem 23 (1999), 469-477.

[14] G. Caporossi and P. Hansen, Variable neighborhood search for extremal graphs 1. The Autographix system, Discrete Math 212 (2000), 29-44.

[15] G. Caporossi and P. Hansen, Variable neighborhood search for extremal graphs 5. Three ways to automate finding conjectures, Discrete Math 276 (2004), 81-94.

[16] T. Christof, PORTA—A POlyhedron Representation Transformation Algorithm, webpage. Version 1.3.2 (1999), written by T. Christof, maintained by A. Loebel and $\mathrm{M}$. Stoer, available at http://www.informatik.uni-heidelberg. de/groups/comopt/software/PORTA/. 
[17] L. Collatz and U. Sinogowitz, Spektren endlicher Grafen, Abh Math Sem Univ Hamburg 21 (1957), 63-77.

[18] D. Cvetković, Discussing graph theory with a computer II: Theorems suggested by the computer, Publ Inst Math (Beograd) 33 (1983), 29-33.

[19] D. Cvetković, Discussing graph theory with a computer IV: Knowledge organisation and examples of theorem proving, Proc 4th Yugoslav Sem Graph Theory, Novi Sad, 1983, pp. 43-68.

[20] D. Cvetković, Discussing graph theory with a computer VI: Theorems proved with the aid of the computer, Cl Sci Math Natur Sci Math 16 (1988), 51-70.

[21] D. Cvetković, L. Kraus, and S. Simić, Discussing graph theory with a computer I: Implementation of graph theoretic algorithms, Univ Beograd Publ Elektrotehn. Fak, Ser Mat Fiz No 716-734 (1981), 100-104.

[22] D. Cvetković and I. Pevac, Discussing graph theory with a computer III: Man-machine theorem proving, Publ Inst Math (Beograd) 34 (1983), 37-47.

[23] D. Cvetković and S. Simić, Graph theoretical results obtained by the support of the expert system "graph", Bull Acad Serbe Sci Arts Cl Sci Math Natur 19 (1994), 19-41.

[24] D. Cvetković, S. Simić, G. Caporossi, and P. Hansen, Variable neighborhood search for extremal graphs 3 . On the largest eigenvalue of color-constrained trees, Linear Multilinear Algebra 2 (2001), 143-160.

[25] E. De la Vina, Bibliography on conjectures of graffiti, Available at: http://cms.dt.uh.edu/faculty/delavinae/research/ wowref.htm, 2000.

[26] S. Fajtlowicz, Written on the wall, A regularly updated file accessible from http://www.math.uh.edu/ clarson/.

[27] S. Fajtlowicz, On conjectures of graffiti II, Congr Numer 60 (1987), 187-197.

[28] S. Fajtlowicz, On conjectures of graffiti, Discrete Math 72 (1988), 113-118.

[29] S. Fajtlowicz, On conjectures of graffiti III, Congr Numer 66 (1988), 23-32.

[30] S. Fajtlowicz, On conjectures of graffiti IV, Congr Numer 70 (1990), 231-240.

[31] S. Fajtlowicz, On conjectures of graffiti V, 7th Int Quadrennial Conf Graph Theory, Vol. 1, 1995, pp. 367-376.

[32] P. Fowler, P. Hansen, G. Caporossi, and A. Soncini, Polyenes with maximum homo-lumo gap, Chem Phys Lett 342 (2001), 105-112.
[33] K. Fukuda, cdd/cdd+ reference manual, webpage. cdd ver. 0.61, cdd+ ver. 0.76 (1999), available at http://www.cs. mcgill.ca/ $\sim$ fukuda/soft/cddman/cddman.html.

[34] I. Gitler and C. Valencia, Bounds for graph invariants. ArXir Mathematics e-prints math/0510387.

[35] I. Gutman, P. Hansen, and H. Mélot, Variable neighborhood search for extremal graphs 10 . Comparison of irregularity indices for chemical trees, J Chem Inf Model 45 (2005), 222-230.

[36] I. Gutman, P. Hansen, and H. Mélot, Variable neighborhood search for extremal graphs 12. A note on the variance of bounded degrees in graphs, MATCH Comm Math Comput Chem 54 (2005), 221-232.

[37] P. Hansen, Bornes et algorithmes pour les stables d'un graphe, A look at graph theory (Proc. Colloq., Cerisy, 1980) (french), Presses Polytech. Romandes, Lausanne, 1980, pp. 39-53.

[38] P. Hansen, How far is, should and could be conjecture-making in graph theory an automated process? Graphs Discovery, Vol. 69 of DIMACS Series in Discrete Mathematics and Theoretical Computer Science, American Mathematical Society, Providence, R.I., 2005, pp. 189-229.

[39] P. Hansen and H. Mélot, Computers and discovery in algebraic graph theory, Linear Algebra Appl 356 (2002), 211-230.

[40] P. Hansen and H. Mélot, Variable neighborhood search for extremal graphs 6. Analysing bounds for the connectivity index, J Chem Inf Comput Sci 43 (2003), 1-14.

[41] P. Hansen and H. Mélot, Variable neighborhood search for extremal graphs 9. Bounding the irregularity of a graph, Graphs Discovery, Vol. 69 of DIMACS Series in Discrete Mathematics and Theoretical Computer Science, American Mathematical Society, Providence, R.I., 2005, pp. 253-264.

[42] J. Harant and I. Schiermeyer, On the independence number of a graph in terms of order and size, Discr Math 232 (2001), 131-138.

[43] B. McKay, Nauty user's guide (version 1.5), Technical report TR-CS-90-02, Department of Computer Science, Australian National University, 1990.

[44] H. Mélot, Facet defining inequalities among graph invariants: The system GraPHedron, Discr Appl Math, in press.

[45] O. Ore, Theory of graphs, American Mathematical Society Colloquium Publications, Vol. 38, American Mathematical Society, Providence, R.I., 1962.

[46] P. Turán, Eine Extremalaufgabe aus der Graphentheorie, Mat Fiz Lapok 48 (1941), 436-452.

[47] G.M. Ziegler, Lectures on polytopes, Vol. 152 of Graduate Texts in Mathematics, Springer-Verlag, New York, 1995. 